\title{
Parâmetros no GeoGebra: Um Estudo sobre a Circunferência
}

\author{
Marciane Linhares Carlos - PPGEMAT/UFRGS \\ marciane.carlos@gmail.com \\ Márcia Rodrigues Notare - PPGEMAT/UFRGS \\ marcia.notare@ufrgs.br
}

\begin{abstract}
Resumo
Este artigo apresenta uma pesquisa realizada com alunos do $3^{\circ}$ ano do Ensino Médio de uma escola da rede privada da grande Porto Alegre, na qual o objetivo foi analisar as contribuições do software GeoGebra no estudo da circunferência, utilizando parâmetros para provocar os alunos a avançar para raciocínios generalizados. O estudo de caso foi utilizado como metodologia e a coleta de dados se deu por meio de observações da professora/pesquisadora, questionamentos sobre as atividades trabalhadas no GeoGebra, registros escritos feitos pelos alunos e dos arquivos .ggb. Neste artigo, serão apresentadas e analisadas duas atividades, nas quais o uso de parâmetros foi utilizado no GeoGebra com o intuito de levar os alunos a compreenderem os elementos da equação da circunferência, generalizar o raciocínio e reconhecer as representações gráfica e algébrica do mesmo objeto matemático.
\end{abstract}

Palavras-chave: circunferência, GeoGebra, parâmetros, generalização

\section{Parameters in GeoGebra: A Study of the circumference}

\begin{abstract}
This article presents a survey of students of the 3rd year of high school to a private network school of the great Porto Alegre, in which the aim was to analyze the GeoGebra software contributions in the study of the circle, using parameters to cause students to advance to generalized reasoning. The case study was used as methodology and the data collection was carried out through observations of teacher/researcher, questions about the activities worked in GeoGebra, written records made by students and.ggb files. In this article, we will be presented and analyzed two activities in which the use of parameters was used in GeoGebra in order to bring students understand the elements of the equation of the circle, generalizing the reasoning and recognize the graphic and algebraic representations of the same mathematical object.
\end{abstract}

Keywords: circumference, GeoGebra, parameters, generalization

\section{Introdução}

O trabalho aqui apresentado é resultado de uma pesquisa realizada com alunos do $3^{\circ}$ ano do Ensino Médio, no qual o objetivo foi investigar as contribuições do GeoGebra no estudo da Geometria Analítica, em específico no estudo da circunferência, utilizando parâmetros para chegar a raciocínios generalizados. Para conduzir a pesquisa, foi desenvolvido um website com as atividades propostas durante a coleta de dados e com as produções finais dos alunos. Neste artigo, serão apresentadas e analisadas duas atividades que envolvem o raciocínio generalizador da equação da circunferência utilizando o GeoGebra.

A pesquisa realizada teve como metodologia o estudo de caso, baseado nos estudos de Ponte (2006) e, comumente utilizado nas investigações em Educação Matemática. Para entender como se dá o processo de aprendizagem da equação da circunferência, utilizando parâmetros que buscam generalizar coleções de circunferências, apresentamos a 
fundamentação teórica, na qual estudamos: o raciocínio generalizador a partir dos estudos de Dreyfus (1991) e da Teoria dos Três Mundos de Tall (2004), no qual a generalização faz parte do terceiro mundo chamado de "Mundo formal axiomático"; e a utilização de parâmetros baseado nos estudos de Notare e Gravina (2013) e Notare et al. (2015).

\section{Fundamentação Teórica: Raciocínio generalizador}

Em seus estudos, Tall (2004) apresenta três tipos distintos de desenvolvimento cognitivo relacionado ao pensamento matemático, os quais fazem parte da sua teoria dos Três Mundos da Matemática: "mundo conceitual corporificado", "mundo operacional simbólico" e "mundo formal axiomático".

Segundo Tall (2004), o primeiro mundo, chamado de "mundo conceitual corporificado", está relacionado com as percepções e as ações que estão ligadas a objetos físicos que possam ser manipulados e depois representados como objetos mentais. Nesta etapa de desenvolvimento, a percepção, a visualização, a observação e a descrição demonstram o entendimento de propriedades dos objetos estudados relacionados aos conceitos matemáticos.

No segundo mundo, denominado "mundo operacional simbólico", Tall (2004) descreve o mundo dos símbolos usados para o cálculo, em manipulações na aritmética e na álgebra e que representam as percepções e ações do mundo corporificado. Todo símbolo tem o que Gray e Tall (1994) chamam de "proceito", que são símbolos que representam tanto um processo como um conceito.

O último mundo, que Tall (2004) chamou de "mundo formal axiomático", faz uso das corporificações e dos proceitos, no qual é necessário formalizar o entendimento do objeto estudado. Ao formalizar, busca-se a especificação de suas propriedades bem como de suas relações, que exige um grau maior de abstração e dos subprocessos da abstração: representação, generalização e síntese.

Para Tall (2004), o desenvolvimento do pensamento matemático vai do mundo conceitual ao mundo formal, ou seja, transita do pensamento matemático elementar ao pensamento matemático avançado, onde cada indivíduo tem a sua trajetória e desenvolvimento individual ao percorrer os Três Mundos da Matemática, sendo o terceiro mundo o que mais exige dos alunos, pois é nele que se busca a formalização, a abstração e os processos que servem de base para a abstração: generalização, síntese e representação.

A abstração, segundo Dreyfus (1991), é acima de tudo um processo construtivo, que implica na construção de estruturas mentais de estruturas matemáticas, ou ainda, que implica na relação entre objetos matemáticos e suas respectivas propriedades.

Dreyfus (1991) entende que o processo de abstração está ligado ao processo de generalização, embora a natureza do processo mental de abstração seja diferente da natureza do processo mental da generalização, pois a "generalização geralmente envolve uma expansão da estrutura de conhecimento do indivíduo enquanto abstração é suscetível de acarretar uma reconstrução mental" (Dreyfus, 1991, p. 36, tradução nossa). O autor ainda comenta que, embora as exigências cognitivas no processo de generalização tenham aumentado bastante ao longo das décadas, o processo de abstração ainda requer uma demanda cognitiva maior por parte do aluno.

Tall (1991), ao usar os termos generalização e abstração na matemática, justifica que é para "denotar os processos em que os conceitos são vistos em um contexto mais amplo e também os produtos desses processos" (Tall, 1991. p. 11, tradução nossa). É preciso perceber que estes dois termos são, na verdade, dois objetos mentais produzidos de acordo com os processos cognitivos envolvidos. 
Quanto à generalização, Dreyfus (1991) define como uma derivação de um caso particular, onde o objetivo é identificar pontos comuns para então expandir os domínios de validação e assim passar de casos particulares para um caso geral.

Neste trabalho, exploramos processo de generalização a partir do uso de parâmetros na equação da circunferência, com o auxílio do dinamismo do software GeoGebra.

\subsection{Utilizando Parâmetros no GeoGebra}

A utilização de parâmetros nos softwares matemáticos vem sendo estudado por Notare e Gravina (2013) e Notare et al. (2015), ao explorarem o potencial semiótico de um software matemático.

Segundo Notare e Gravina (2013, p. 13), "a utilização do potencial de um software depende muito do entendimento que se tem das representações semióticas que nele se tem a disposição", ou seja, quanto mais se conhece as ferramentas disponíveis no software e suas funcionalidades, mas tem-se a oportunidade de explorar o potencial semiótico desta ferramenta em suas diferentes representações.

O objetivo de utilizar parâmetros é de poder generalizar um padrão que se repete, podendo assim, estimular a compreensão de maneira global do objeto estudado, pois

O trabalho com parâmetros exige dos alunos uma compreensão mais global das relações matemáticas e seus coeficientes, uma vez que os alunos precisam trabalhar, simultaneamente, com os registros algébrico e gráfico e controlar estes parâmetros no registro algébrico para obter o efeito gráfico desejado no registro gráfico. (Notare et al., 2015, p.12)

Desta forma, percebemos que o comando sequência do GeoGebra poderia contribuir para o trabalho com generalizações, uma vez que permite representar famílias de formas geométricas a partir de somente uma equação, variando seus parâmetros. Além disso, observar, de forma simultânea, as representações gráfica e algébrica da circunferência, o que possibilita uma compreensão mais ampla e profunda do objeto de estudo. Para compreender o funcionamento do comando sequência, faz-se necessário o entendimento dos significados matemáticos através do conjunto de relações entre signos e seus significados que serão utilizados.

Na equação da circunferência, $(x-a)^{2}+(y-b)^{2}=r^{2}$, tem-se o conjunto de signos $\left\{\mathrm{a}, \mathrm{b}, \mathrm{r}^{2}, \mathrm{x}, \mathrm{y},-,=\right\}$, as letras $a, b$ e $r$ (são os parâmetros da equação), $x$ e $y$ (são as variáveis), nas relações entre signos e significados, neste caso temos $(\mathrm{x}, \mathrm{y})$ sendo um par ordenado como um ponto qualquer na circunferência; $(\mathrm{a}, \mathrm{b})$ o par ordenado que representa o centro da circunferência e $r$ a medida do raio da circunferência, onde a representação algébrica $(x-a)^{2}+(y-b)^{2}=r^{2}$ apresenta uma circunferência que pode ser mostrada geometricamente no plano cartesiano.

Para exemplificar o uso do comando sequência, criar-se-á uma família de quatro circunferências tangentes externas, na horizontal, de mesmo raio. Para tanto, estipular-se-á que todas as ordenadas do centro das circunferências (representadas pelo signo $b$ ) serão iguais e será atribuído o valor 1, assim como os raios de todas as circunferências (representados pelo signo $r$ ) serão iguais e será atribuído o valor 2. Para construir a família de circunferências, no campo de entrada do GeoGebra, digita-se sequência e escolhe-se a opção ilustrada na Figura 1 . 


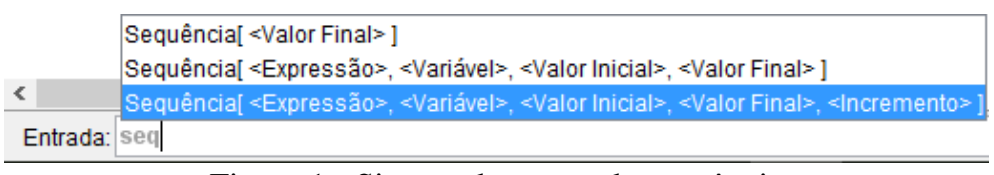

Figura 1 - Sintaxe do comando sequência

Em Expressão, digita-se a equação da circunferência que será criada;

Em Variável, digita-se a variável que deve mudar seu valor para gerar a sequência de circunferências;

Em Valor Inicial, digita-se o valor inicial que a Variável deve assumir;

Em Valor Final, digita-se o valor final que a Variável deve assumir;

Em Incremento, digita-se o valor que a Variável deve propagar.

Aplicando os valores do exemplo, o campo entrada ficará como ilustrada na Figura 2.

Entrada: Sequência[(x-a)^2+(y-1)^2=2^2, a, 2, 14, 4]

Figura 2 - Sintaxe do comando sequência com os seus respectivos valores

Nesse caso, tem-se primeiramente a equação da circunferência com a ordenada do centro e o raio definidos, e a abscissa do centro sendo a variável $a$; na sequência estipular-se-á quem será a variável, no caso $a$; vindo seguido dos valores inicial e final que esta variável irá assumir, ou seja, a primeira circunferência terá abscissa do centro igual a 2 e a última circunferência terá abscissa do centro igual a 14. Para garantir que estas circunferências sejam tangentes externas, as abcissas dos centros das circunferências formarão uma progressão aritmética de razão 4 , pois sendo o raio das circunferências igual a $2, \log o$ o diâmetro será 4 e, portanto, tem-se o incremento igual a 4, garantindo assim que todas as circunferências sejam tangentes externas.

Depois de digitada a sequência da Figura 2, o GeoGebra gera, na janela de álgebra, uma lista com todas as equações das circunferências criadas a partir da generalização do comando sequência e, simultaneamente, na janela de visualização, a representação geométrica desta família de circunferências, como mostra a Figura 3.

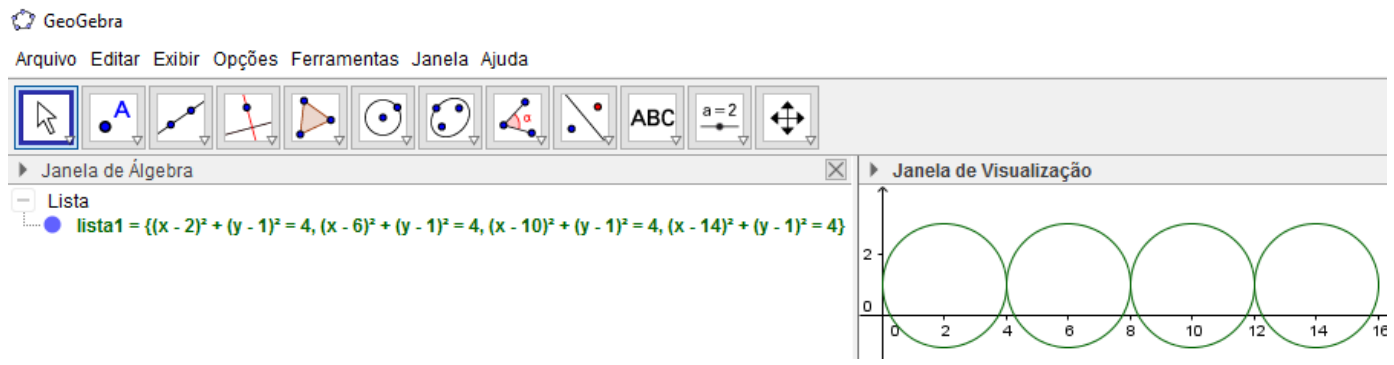

Figura 3 - Representações algébrica e geométrica a partir do comando sequência

Existem estudos no meio acadêmico sobre o uso de parâmetros no GeoGebra, como o de Silva (2013) que, em seu trabalho, explora o estudo de várias funções por meio da variação de parâmetros, cujo objetivo é fazer com que os alunos aprendam a identificar e descrever os efeitos da variação de parâmetros nas funções elementares, compreendendo assim o comportamento gráfico das funções e o papel desempenhado por cada um dos coeficientes das relações algébricas. Diferente da pesquisa aqui apresentada, a utilização de parâmetros no trabalho de Silva (2013) não tem como objetivo estudar a generalização, criando família de funções, e sim o comportamento gráfico das funções ao serem modificados os parâmetros. A parametrização e ao raciocínio generalizador são utilizados nas pesquisas de Notare e Gravina 
$(2013,2015)$ ao abordarem o assunto de Geometria Analítica por meio da réplica de obras de arte e da ilusão de óptica, porém em outro software, o GrafEq.

A seguir, apresentamos duas atividades da pesquisa e analisamos o avanço dos alunos na compreensão da circunferência.

\section{Atividades no GeoGebra - Explorando parâmetros}

As atividades práticas deste trabalho foram aplicadas em uma escola particular da região metropolitana de Porto Alegre, onde a pesquisadora deste trabalho é professora de Matemática do Ensino Médio, em uma turma do $3^{\circ}$ ano do Ensino Médio que, no início da pesquisa, contava com dezessete alunos e, no decorrer da pesquisa, chegaram mais dois alunos novos nesta turma. Os alunos fizeram as atividades em duplas e um trio, formando assim oito grupos. Ao todo, foram utilizados catorze períodos de 45 minutos para aplicação das atividades. Nesta pesquisa, foram analisados quatro grupos (A, C, E e H) que, diferente dos demais grupos, compareceram em todos as aulas e, desenvolveram e entregaram todas as atividades.

A metodologia utilizada foi o Estudo de Caso e teve como referência os estudos de Ponte (2006). Foi escolhida por se tratar de uma investigação em Educação Matemática, que tem como propósito estudar uma situação específica e que, segundo Ponte (2006), o objetivo da investigação num estudo de caso é entender e compreender o "como" e os "porquês" de uma entidade que pode ser uma pessoa, instituição, disciplina na área da educação ou de outra área de conhecimento.

Ponte (2006) acredita que os estudos de casos podem ter alguns propósitos bem específicos, como: ser exploratório e servir para obter informações preliminares; ser descritivo e relatar como é o caso estudado; e ser analítico construindo ou desenvolvendo uma nova teoria ou então confrontar com uma que já existe.

A coleta de dados se deu por meio de questionamentos sobre as atividades que estavam sendo trabalhadas no GeoGebra, registros escritos feitos pelos alunos, observações da professora/pesquisadora, e arquivos do GeoGebra.

Para atender os objetivos deste trabalho, foi elaborada uma sequência de dez atividades disponibilizadas em um website (http://marcianecarlos.wix.com/matematica). Na primeira aula, após ser apresentado o website para os alunos, foi acordado que todas as circunferências deveriam ser construídas pelo Campo de Entrada do GeoGebra, para provocar a elaboração da equação da circunferência. Destas atividades, serão apresentadas e analisadas duas, nas quais os parâmetros foram utilizados no GeoGebra com o intuito de generalizar o raciocínio em torno do estudo da circunferência.

\subsection{Atividade A: Construir sequência de circunferências}

Nesta atividade o objetivo foi explorar o comando sequência, no qual se utilizou parâmetros da equação da circunferência para, a partir de uma equação, criar a representação geométrica de uma família de circunferências, conforme Figura 4.

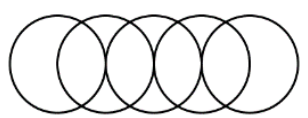

Figura 4 - Família de circunferências

Para tanto, os alunos precisavam identificar quais parâmetros da equação da circunferência deveriam variar para resultar na construção da Figura 4. Esse processo inicia na análise da representação gráfica da família de circunferências, para identificar os elementos 
da representação algébrica que precisam se manter constantes e os elementos que precisam variar e como eles devem variar. Antes dos alunos partirem para a construção da família de circunferências, deveriam responder duas perguntas sobre parâmetros com base na Figura 4:

1. Pensando na equação da circunferência para criar esta sequência de circunferências, $o$ que você acha que deve variar de uma circunferência para outra da sequência?

2. Como podemos criar uma sequência de circunferências conforme a figura acima?

Para responderem às duas questões, dois grupos, $\mathrm{C}$ e $\mathrm{H}$, não conseguiram, de imediato, identificar quais parâmetros da equação permanecem constantes e quais devem variar, o que revela, neste momento, ainda uma falta de compreensão da circunferência e as relações existentes entre os registros gráfico e algébrico. Para solucionar o problema, os alunos construíram cinco circunferências semelhantes às circunferências propostas pela atividade e, a partir da observação de suas equações, constataram que o parâmetro variável na equação era a abscissa do centro, conforme ilustra a Figura 5.

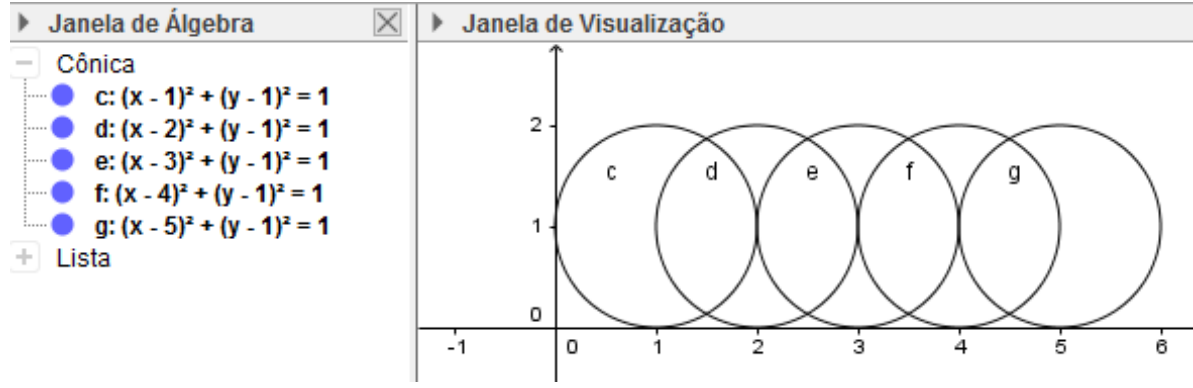

Figura 5 - Construção feita pelo grupo $\mathrm{H}$

Percebe-se, neste caso, a necessidade dos alunos em apoiarem-se nos recursos do software para solucionar a questão, sendo ainda incapazes de antecipar e estabelecer os valores para centro e raio da equação a partir da representação gráfica.

Os alunos do grupo A criaram controles deslizantes para o centro e o raio, com o intuito de explorarem e visualizarem o que estava variando e, a partir desta exploração, concluíram que a abscissa do centro deveria ser variável, como mostra a Figura 6.

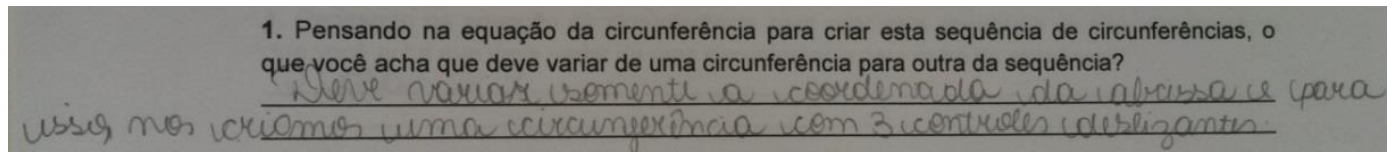

Figura 6 - Resposta do grupo A ao item 1

Nota-se que estes alunos estão utilizando o GeoGebra como uma ferramenta para pensar em Matemática e para auxiliar no processo de reconhecimento e generalização da equação da circunferência.

A dupla E também utilizou esta estratégia para solucionar o problema, mas com dificuldades para determinar os valores para centros e raios. Dessa forma, estes alunos tentaram encontrar os valores por tentativa e erro, até chegarem ao resultado esperado. Isso revela que estes alunos se encontram em processo de exploração e descoberta sobre a equação da circunferência e sua representação gráfica.

Ao final da atividade, todos os grupos conseguiram construir a sequência de circunferências propostas (Figura 4) a partir do comando sequência, com exceção do grupo A, 
que digitou um valor específico para a abscissa do centro, ao invés de utilizar um parâmetro. Embora soubessem, quando questionados, que era a abscissa do centro que estava variando, pensaram que não poderiam digitar uma variável, conforme os alunos explicaram nos seus registros (Figura 7).

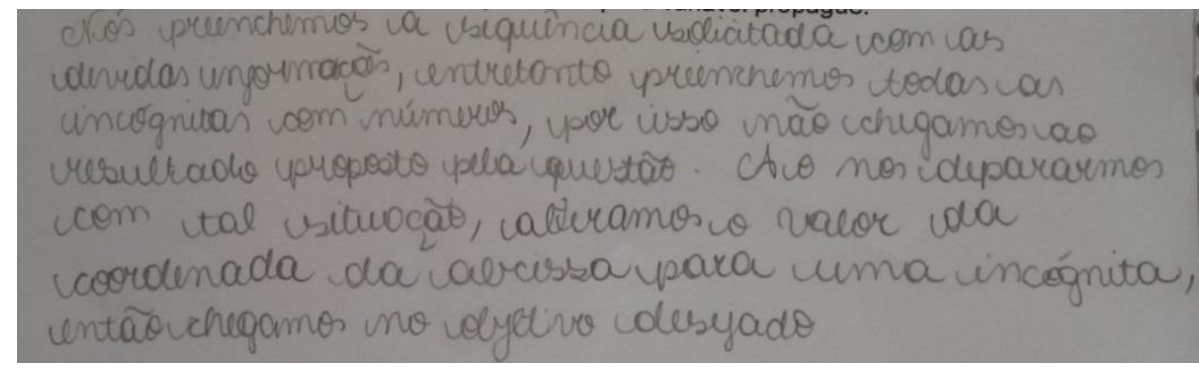

Figura 7 - Explicação do grupo A sobre como construíram a família de circunferências

Percebe-se na escrita do grupo A que a ideia de generalizar criando uma variável que represente um parâmetro ainda não havia sido consolidada. Ao preencher "todas as incógnitas com números", os alunos referem-se à Figura 2, na qual deveriam completar a sintaxe do comando sequência com os seus respectivos valores.

\subsection{Atividade B: Construir sequências das posições relativas entre circunferências}

Nesta atividade, o objetivo foi construir sequências de posições relativas entre circunferências a partir das suas equações e utilizando o pensamento generalizador. A partir de uma representação gráfica de sequências de circunferências, foi preciso encontrar uma expressão algébrica que a generalize, levando ao entendimento mais global da circunferência. As questões solicitadas estão apresentadas a seguir:

1.

a) Crie, no GeoGebra, uma sequência de circunferências externas a partir de uma única relação.

b) Como você pensou para criar esta sequência de modo a garantir que são externas?

c) Analisando a expressão criada para a sequência de circunferências externas, o que você mudaria na expressão para torná-las circunferências secantes?

2.

a) Crie, no GeoGebra, uma sequência de circunferências tangentes externas a partir de uma única relação.

b) Como você pensou para criar esta sequência de modo a garantir que são tangentes externas?

c) Analisando a expressão criada para a sequência de circunferências tangentes externas, o que você mudaria na expressão para torná-las circunferências concêntricas?

No item 1, o grupo A criou circunferências tangentes externas ao invés de circunferências externas, mas perceberam que, mudando o incremento, poderiam torná-las externas, conforme registro feito pelo grupo: Digitamos incógnitas na fórmula da circunferência de maneira que as mesmas pudessem ser alteradas mas na primeira vez deu errado pois a distância entre os centros eram iguais a soma dos dois raios, por isso alteramos o incremento para dar certo. A primeira vez que deu errado ficou uma tangente externa. Percebe-se que os alunos conseguiram compreender as relações relativas das circunferências quando visualizaram a representação gráfica da sequência de circunferências 
criadas, mostrando a importância da manipulação do objeto estudado nas diferentes representações que o software proporciona. Percebe-se também que o grupo conseguiu generalizar em uma única expressão algébrica a sequência de circunferências e que fizeram uso da manipulação dos parâmetros para chegarem ao resultado desejado.

O grupo C, ao responder o item 1.b), perguntou se era para usar o comando sequência como na atividade anterior, pediu-se para lerem novamente o enunciado e concluíram sozinhos que deveriam utilizar o comando sequência para a construção das circunferências, e o grupo colocou a expressão generalizada que criou as circunferências externas, conforme Figura 8. A Figura 9 ilustra as circunferências construídas no GeoGebra.

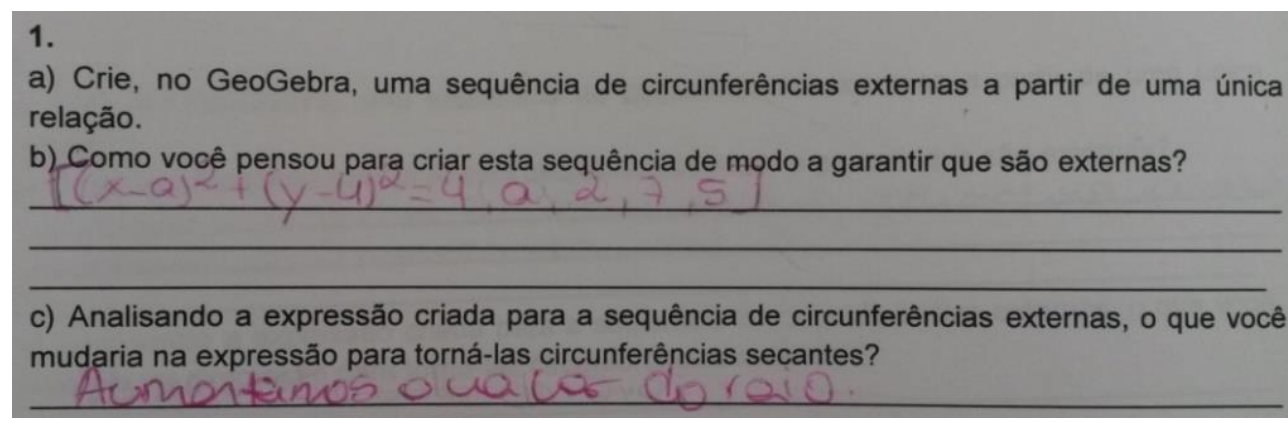

Figura 8 - Item 1 respondido pelo grupo C
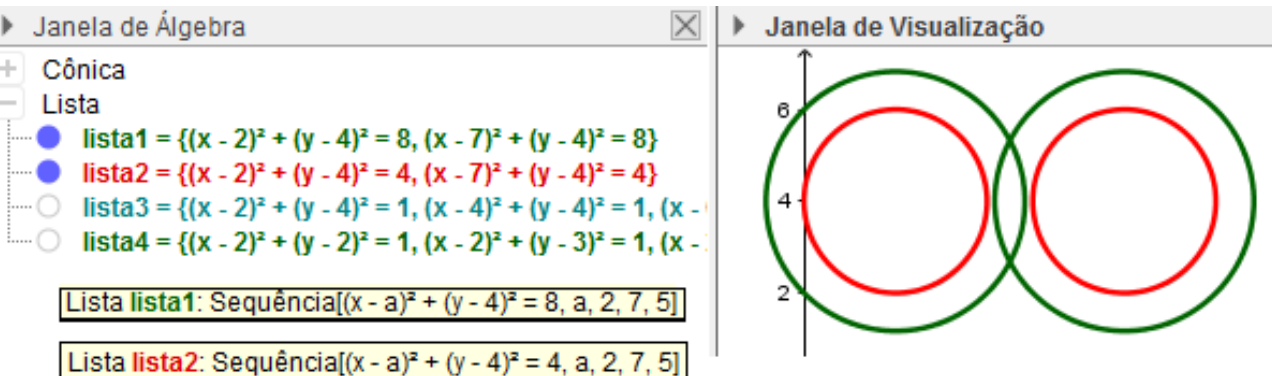

Figura 9 - Circunferências construídas pelo grupo C a partir da solicitação do item 1

Nota-se que, embora sem saber como começar a atividade B por falta de atenção, os alunos transformaram as circunferências externas (na Figura 9 na cor vermelha) em circunferências secantes (na Figura 9 na cor verde) mudando apenas o valor do raio, optando assim por não alterar o parâmetro $a$, conforme mostram as listas 1 e 2 da Figura 9.

A dupla $\mathrm{H}$ construiu várias circunferências a partir das equações e não sabia como construir uma única relação para as várias circunferências. Ao serem lembrados do comando sequência, construíram as circunferências, porém secantes ao invés de externas como pedia a atividade. Foi preciso manipular os parâmetros para obter as circunferências secantes.

No item 2, que solicitava a construção de uma sequência de circunferências tangentes externas para depois torná-las concêntricas, o grupo A teve dificuldades em tornar a sequência de circunferências tangentes externas em concêntricas, como ilustrado na Figura 10. 


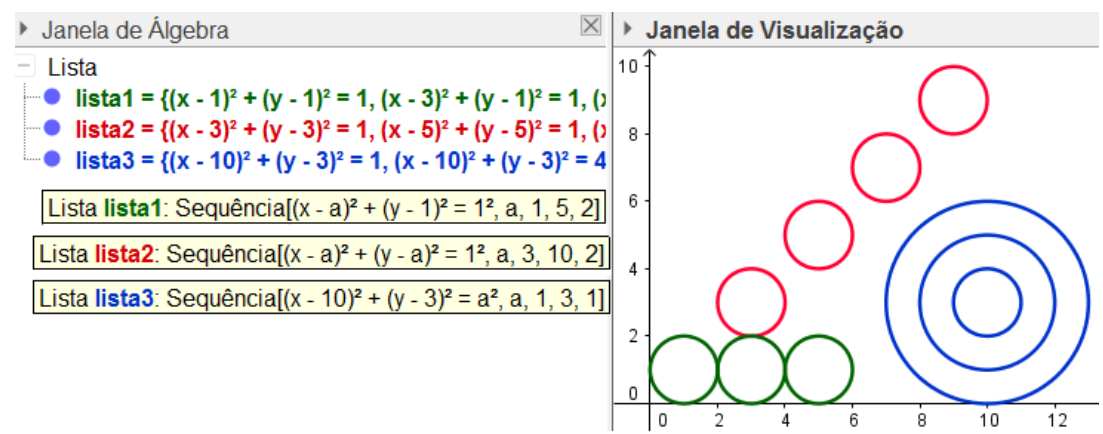

Figura 10 - Item 2 desenvolvido pelo grupo A

O grupo A iniciou seu raciocínio corretamente, ao utilizar o parâmetro $a$ para determinar os valores para a abscissa dos centros das circunferências ao criar a lista 1, que gerou a coleção de circunferências tangentes externas ilustradas na Figura 10 na cor verde. No momento de transformar esta coleção de circunferências tangentes externas em circunferências concêntricas, os alunos deste grupo geraram uma sequência de circunferências distribuídas em diagonal, como mostra a Figura 10 na cor vermelha. Isso ocorreu porque utilizaram os parâmetros para determinar as coordenadas do centro da circunferência (Figura 10, lista 2). Ao visualizarem a representação gráfica da sequência que criaram, perceberam que os centros das circunferências deveriam ter um valor fixo. Ao alterarem apenas o valor das coordenadas dos centros, os alunos geraram circunferências sobrepostas, pois todos os raios estavam com medidas iguais. Ao serem questionados sobre como visualizaram todas as circunferências sobrepostas, perceberam que o parâmetro deveria ser usado para variar as medidas de raio, e assim, avançaram para a expressão representada na lista 3 (Figura 10), que gerou a coleção de circunferências concêntricas ilustradas na Figura 10 na cor azul. $O$ grupo A precisou apoiar-se na representação gráfica da coleção de circunferências para compreender o papel de cada elemento da representação algébrica e, assim, utilizar os parâmetros de forma a gerar a sequência desejada. Esse processo auxilia os alunos na compreensão do objeto circunferência de forma mais global, pois exige raciocínio sobre diferentes representações.

A dupla $\mathrm{H}$ não estava conseguindo construir as circunferências concêntricas, pois estava com a abscissa do centro variando. Estes alunos conseguiram perceber o parâmetro que deveriam alterar quando questionados, conforme diálogo:

- O que vocês querem que fique variando? - professora/pesquisadora.

- $O$ raio. - aluno da dupla $H$.

- Mas o que está variando? - professora/pesquisadora.

- Ah, a gente deve alterar a variável para o raio. - aluno da dupla $H$.

Esperava-se que, ao lerem o enunciado da atividade, percebessem que deveriam utilizar a generalização (embora em nenhum momento foi falado no pensamento generalizador), já utilizada na atividade anterior. Porém, alguns alunos estavam inseguros e questionaram a maneira sobre como fazer; outros, só utilizaram o comando sequência quando foi percebido que estavam criando uma a uma as circunferências. Os alunos conseguiram compreender as relações das circunferências quando visualizaram a representação gráfica da sequência de circunferências criadas, mostrando a importância da manipulação do objeto estudado no GeoGebra em suas diferentes representações, e a importância de entender o significado dos parâmetros na expressão algébrica generalizada. 


\section{Considerações Finais}

Ao analisar as atividades propostas, nota-se que os alunos utilizaram o GeoGebra como um recurso para pensar em Matemática, para explorar o objeto matemático circunferência em suas diferentes representações. A partir da manipulação no GeoGebra, os alunos identificaram pontos que estavam variando em uma família de circunferências e os representaram por meio de uma variável, para conseguir expressar estas circunferências em uma única expressão algébrica, ou seja, fizeram uso dos parâmetros para alcançarem, na atividade cognitiva, a generalização, que segundo Tall (2004) faz parte do terceiro mundo, o mundo formal axiomático.

Percebe-se o quão importante foi o uso da tecnologia, o GeoGebra, nesta experiência para os alunos conseguirem transitar entre os Três Mundos da Matemática ao perceberem, visualizarem, observarem, descreverem e formalizarem as propriedades das circunferências e suas relações.

\section{Referências}

DREYFUS, Tommy. Advanced Mathematical Thinking Processes. In: Tall, David (Ed.), Advanced Mathematical Thinking. Norwell: Kluver Academic Publishers, 1991, p. 25 - 41.

GRAY, E. \& TALL, D. O.: (1994). Duality, Ambiguity and Flexibility: A Proceptual View of Simple Arithmetic, The Journal for Research in Mathematics Education, 26 (2), 115-141.

NOTARE, M. R.; FIOREZE, L.A. ; HALBERSTADT, F. F. . O Software Grafeq e os Registros de Representação Semiótica: Uma Análise de Trabalhos com Ilusão de Ótica. In: XIV Conferencia Interamericana de Educação Matemática - XIV CIAEM, 2015, Tuxtla. XIV Conferencia Interamericana de Educación Matemática, 2015.

NOTARE, M.R., GRAVINA, M. A. (2013). A Formação Continuada de Professores de Matemática e a Inserção de Mídias Digitais na Escola. Anais do VI Colóquio de História e Tecnologia no Ensino de Matemática (VI HTEM), São Carlos, SP, Brasil. Disponível em: http://htem2013.dm.ufscar.br/anais/artigoscompletos/artigocompleto_OC_T1_13_MarciaNota re_MariaAliceGravina_versao_final.pdf. Acesso em 15 jun 2016.

PONTE, J. P. (2006). Estudos de caso em educação matemática. Bolema, 25, 105-132. Este artigo é uma versão revista e actualizada de um artigo anterior: Ponte, J. P. (1994). O estudo de caso na investigação em educação matemática. Quadrante, 3(1), 3-18. (re-publicado com autorização).

SILVA, Luís Gustavo. Variação de Parâmetros em Funções Elementares Utilizando o GeoGebra. Dissertação de Mestrado. UFTM, 2013. Disponível em: http://bit.profmatsbm.org.br/xmlui/handle/123456789/421. Acesso em 04 nov 2016.

TALL, David. The Psychology of Advanced Mathematical Thinking. In: Tall, David (Ed.), Advanced Mathematical Thinking. Norwell: Kluver Academic Publishers, 1991, p. 3 - 21.

TALL, David (2004). Introducing Three Worlds of Mathematics. For the Learning of Mathematics, 23 (3). 29-33. 